\title{
Direct observation of strong correlations near the band insulator regime of Bi misfit cobaltates
}

\author{
V. Brouet, ${ }^{1}$ A. Nicolaou, ${ }^{1}$ M. Zacchigna, ${ }^{2}$ A. Tejeda, ${ }^{3}$ L. Patthey, ${ }^{4}$ \\ S. Hébert, ${ }^{5}$ W. Kobayashi, ${ }^{5}$ H. Muguerra, ${ }^{5}$ and D. Grebille ${ }^{5}$ \\ ${ }^{1}$ Laboratoire Physique des Solides, UMR 8502, CNRS, Université Paris-Sud, Bâtiment 510, 91405 Orsay, France \\ ${ }^{2}$ CNR-INFM, Laboratorio Nazionale TASC, clo Area Science Park, s.s. 14 Km. 163.5, I-34012 Basovizza (TS), Italy \\ ${ }^{3}$ Laboratoire Matériaux et Phénomènes Quantiques, UMR 7162, CNRS, Université Paris Diderot, \\ Boîte Postale 7021, 75205 Paris, France \\ ${ }^{4}$ Swiss Light Source, Paul Scherrer Institut, CH-5234 Villigen, Switzerland \\ ${ }^{5}$ Laboratoire CRISMAT, UMR 6508, CNRS et Ensicaen, 14050 Caen, France
}

(Received 18 June 2007; revised manuscript received 31 July 2007; published 10 September 2007)

\begin{abstract}
We establish with angle-resolved photoemission spectroscopy (ARPES) that the electronic structure near the Fermi level of the "misfit" cobaltate $\left[\mathrm{Bi}_{2} \mathrm{Ba}_{2} \mathrm{O}_{4}\right] \cdot\left[\mathrm{CoO}_{2}\right]_{2}$ is similar to that of $\mathrm{Na}$ cobaltates $\mathrm{Na}_{x} \mathrm{CoO}_{2}$. Both families of compounds contain triangular Co planes but in a different three-dimensional environment. We propose that the peculiar ARPES line shape of all cobaltates is of the "peak-dip-hump" type, due to strong many-body effects. We detect a progressive transfer of spectral weight from the quasiparticle feature near $E_{F}$ to a broad hump in misfit phases where $\mathrm{Ba}$ is replaced by $\mathrm{Sr}$ or $\mathrm{Ca}$. This indicates stronger many-body interactions in proximity to the band insulator regime, which we attribute to the presence of unusual magnetic excitations.
\end{abstract}

DOI: 10.1103/PhysRevB.76.100403

Describing the motion of holes on a triangular Co lattice is a fundamental challenge, because of the interplay between many complex phenomena. Strong correlations coexist with magnetic frustration, orbital degeneracy, and possibly charge-ordering effects. This situation occurs in $\mathrm{Na}$ and misfit cobaltates, where a triangular array of $\mathrm{Co}$ is embedded in edge-sharing oxygen octahedra, forming $\mathrm{CoO}_{2}$ slabs. Paradoxically, metallic phases in $\mathrm{Na}_{x} \mathrm{CoO}_{2}$ exhibit apparently simple Pauli susceptibility at $x<0.5$ and Curie-Weiss $(\mathrm{CW})$ susceptibility and magnetic orders at $x>0.7 .{ }^{1}$ One would rather have expected stronger magnetic correlations near the Mott insulator (expected at $x=0$, where each site would be $\mathrm{Co}^{4+}$ with spin $\left.S=1 / 2\right)$ than near the band insulator $(x=1$, with only nonmagnetic $\mathrm{Co}^{3+}$ ). This unexpected contrast clearly calls for an explanation. It was sometimes assigned to the role of $\mathrm{Na}$, whose potential may localize electrons on some Co sites in Na-rich phases. ${ }^{2,3}$ However, misfit cobaltates, which are Na-free, also exhibit metallic phases with weak to strong $\mathrm{CW}$ behaviors ${ }^{4-6}$ and even a progressive localization of carriers near the band insulator regime ${ }^{5,6}$ not observed in Na phases. ${ }^{7}$ In addition, both families exhibit high thermoelectric powers, ${ }^{4-8}$ with unusual magnetic field dependences. ${ }^{8}$

Angle-resolved phtoemission spectroscopy (ARPES) is a sensitive probe of correlation effects, because removing one electron from a metal in the photoemission process reveals the way it was bound to its environment. To clarify the nature of correlations through the phase diagram of cobaltates, we present an ARPES investigation of three misfit cobaltates, ranging from the metallic to the nearly insulating regime. This study supports the idea that the ARPES line shape of cobaltates cannot be simply understood in terms of band effects, as previously assumed, ${ }^{9}$ but that many-body effects are essential. We propose that the line shape is of the "peak-diphump" (PDH) type, a structure often observed in correlated systems. ${ }^{10}$ A coherent quasiparticle (QP) peak is followed by an incoherent tail, the hump. The QP is an electron dressed by collective excitations, and the hump corresponds to ex- cited states related to this dressing. We observe a transfer of spectral weight from the QP peak to the hump near the insulating regime. This indicates increasing correlation effects, which would be expected near a Mott insulator, but are more surprising here, near the band insulator. As magnetic correlations get stronger in this part of the phase diagram, this strongly suggests that they play an essential role in the dressing of the QP and gives indications to model these excitations.

In $\mathrm{Bi}$ misfit cobaltates, the $\mathrm{CoO}_{2}$ slabs are stacked with four pseudoquadratic rocksalt (RS) layers $A-\mathrm{O} / \mathrm{Bi}-\mathrm{O} / \mathrm{Bi}-\mathrm{O} / A-\mathrm{O}(A=\mathrm{Ba}, \mathrm{Sr}, \mathrm{Ca})$. Their ideal formula is $\left[\mathrm{Bi}_{2} A_{2} \mathrm{O}_{4}\right]^{\mathrm{RS}} \cdot\left[\mathrm{CoO}_{2}\right]_{m}$ but substitutions and/or vacancies are commonly found in the RS. $4,11,12$ The relative periodicity $m$ between RS and triangular layers is usually incommensurate. It increases with the size of $A$ from $m=1.67$ for $\mathrm{Ca}$ to 1.82 for Sr and nearly 2 for $\mathrm{Ba}^{13}$ The RS transfers $x$ electrons to the $\mathrm{CoO}_{2}$ slabs, i.e., there are $(1-x)$ holes in the triply degenerate $\mathrm{Co} t_{2 g}$ band (this definition of $x$ is equivalent to that in $\mathrm{Na}_{x} \mathrm{CoO}_{2}$ ). The value of $x$ cannot be exactly anticipated because of the nonstoichiometries, but, all $A$ being divalent, one expects $x$ to increase as $1 / m$ from $\mathrm{Ba}$ to $\mathrm{Sr}$ to $\mathrm{Ca}$. Our single crystals were prepared by a standard flux method ${ }^{11}$ and characterized by magnetic and transport measurements. ${ }^{14}$ In $\mathrm{BiBaCoO}$, there is a weak $\mathrm{CW}$ contribution to the susceptibility and $d \rho / d T$ is positive down to low temperatures. ${ }^{4}$ For $\mathrm{Sr}$ and $\mathrm{Ca}$, the $\mathrm{CW}$ contribution increases and there is an upturn in resistivity below $50 \mathrm{~K}$ for $\mathrm{Sr}$ (Ref. 5) and $200 \mathrm{~K}$ for $\mathrm{Ca}^{6}{ }^{6,14}$ Previous ARPES investigations reported spectra with small weight at the Fermi level and no angular dependence in $(\mathrm{Bi}, \mathrm{Pb}) \mathrm{SrCoO}$ (Refs. 15 and 16) and a QP peak in $(\mathrm{Bi}, \mathrm{Pb}) \mathrm{BaCoO} .{ }^{17} \mathrm{We}$ present a complete mapping of the Fermi surface (FS) in $\mathrm{BiBaCoO}$ and establish that its electronic structure is very close to that of $\mathrm{Na}$ cobaltates. In $\mathrm{BiSrCoO}$ and $\mathrm{BiCaCoO}$, we show that the spectral weight at the Fermi level is gradually transferred to dispersive structures at high binding energies. This evolution, never ob- 


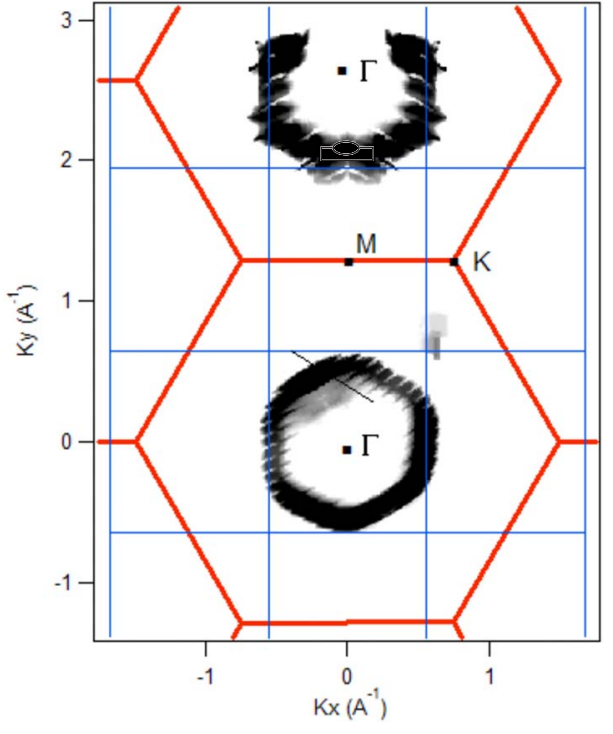

FIG. 1. (Color online) Fermi surface of $\mathrm{BiBaCoO}$ measured at $15 \mathrm{~K}$, obtained by integration of the spectral weight in a $15 \mathrm{meV}$ window around $E_{F}$. Thick red and thin blue lines indicate the Brillouin Zone (BZ) limits for triangular and RS planes. Data have been symmetrized with respect to $k_{y}=-\sqrt{3} k_{x}$ in the first $\mathrm{BZ}$ and $k_{x}=0$ in the second BZ.

served in Na cobaltates, allows us to clarify the line shape structure.

ARPES measurements were carried out at the SIS beamline of the Swiss Light Source (for $\mathrm{BiBaCoO}$ and $\mathrm{BiSrCoO}$ ) and at the BACH beamline of ELETTRA ( $\mathrm{BiCaCoO})$. The photon energy was set near $100 \mathrm{eV}$ and the beam was linearly polarized ( $p$ geometry; see Ref. 9 ). A SCIENTA analyzer was used, with an angular resolution of $0.15^{\circ}$ and a global energy resolution of $15 \mathrm{meV}$. The $\mathrm{FS}$ of $\mathrm{BiBaCoO}$ is presented in Fig. 1. Two hexagons are observed, centered at $(0,0)$ and $\left(0,2.55 \AA^{-1}\right)$. This periodicity matches the one of the triangular plane, and the hexagonal shape of the FS also evidences its connection with the triangular lattice. This establishes that the metallicity is a property of the $\mathrm{CoO}_{2}$ slabs. Hence, the comparison with Na cobaltates is meaningful. In $\mathrm{Na}$ cobaltates, a very similar hexagonal FS is indeed observed ${ }^{18,19}$ where the hexagon corresponds to crossing of the singly degenerate $a_{1 g}$ orbital of the $t_{2 g}$ Co manifold. ${ }^{20} \mathrm{As}$ in Na cobaltates, we do not clearly observe other bands approaching the Fermi level, although local density approximation (LDA) calculations ${ }^{20}$ predict $e_{g}^{\prime}$ bands to form small pockets in the $\Gamma K$ direction.

The FS area is simply related by the Luttinger theorem to the number of holes per Co in the $a_{1 g}$ band. ${ }^{18}$ This gives us a way to estimate the actual hole doping of the Co layers, crucial information in misfit cobaltates, where it cannot be simply deduced from the chemical composition. From measurements in three different samples, we estimate the Fermi wave vector along $\Gamma M$ to be $k_{f}^{(M)}=0.57(5) \AA^{-1}$, leading to $x=0.70(5)$. This value is consistent with the known properties of $\mathrm{BiBaCoO}$, which resemble those of $\mathrm{Na}_{x} \mathrm{CoO}_{2}$ with $x$ $\approx 0.6-0.7$.

We consider in Fig. 2 the electronic structure on a broader
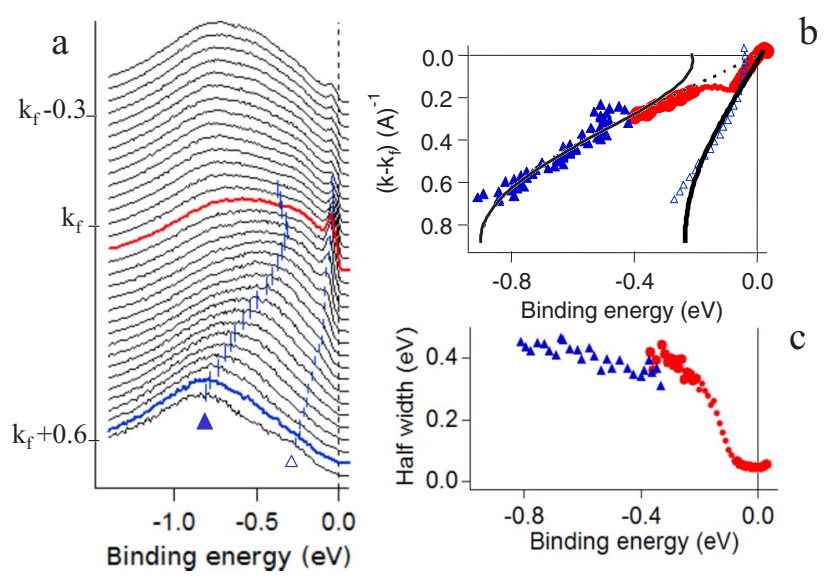

FIG. 2. (Color online) Dispersion in $\mathrm{BiBaCoO}$ along the direction shown in Fig. 1. (a) EDC spectra, spaced by $0.035 \AA^{-1}$, with bars indicating the position of the shoulder (filled triangles) and QP peak (open triangles). (b) Dispersion obtained from MDC fit (red circles) and EDC local maximum (blue triangles). Black lines are cosine fits of these dispersions. (c) Half width at half maximum from MDC (width is normalized by slope of dispersion) and EDC spectra.

energy window. Two dispersing features are resolved, the narrow $a_{1 g}$ QP peak near $E_{F}$ that traces the Fermi surface (open triangle), and a broad shoulder at higher binding energies (filled triangle). They appear on top of a broad peak, centered at $-0.8 \mathrm{eV}$, which corresponds to Co orbitals in the LDA calculation ${ }^{20}$ and which is found with similar width and position in all cobaltates. The direction studied in Fig. 2 is not a high-symmetry one (it is $25^{\circ}$ off $\Gamma K$, as indicated in Fig. 1), but we observe a similar structure in all directions when approaching the FS. ${ }^{21}$ The universality of this feature is also established by the similar data reported in Na cobaltates, for both $\Gamma M$ and $\Gamma K$ directions. ${ }^{9,18}$

In Figs. 2(b) and 2(c), we present a complete analysis of these two features. Near the Fermi level, the spectra at constant energy [momentum distribution curves (MDCs)] can be fitted by a Lorentzian, yielding the QP dispersion indicated by red circles in Fig. 2(b). As the QP peak vanishes, the MDC dispersion follows a nearly horizontal line (smallersize symbols), which is not meaningful and should not be confused with a kink in the dispersion. The QP itself can still be distinguished in the energy distribution curve (EDC) spectra [open blue triangles in Figs. 2(a) and 2(b)] and seems to form a very narrow band of about $200 \mathrm{meV}$. The slope near the Fermi level for this QP dispersion is $V_{\mathrm{QP}}$ $=0.35(5) \mathrm{eV} \AA, 22$ a similar order of magnitude as in Na cobaltates. This is a very small velocity (corresponding to $\mathrm{m}^{*}$ $\left.=13 m_{e}\right)$, but the band mass is itself rather large, ${ }^{20}$ implying $m^{*} \approx 3 m_{\text {band }}$. The MDC fit again tracks correctly the dispersion of the broad shoulder for $E<-0.2 \mathrm{eV}$. It is well aligned with the dispersion extracted from the maximum of the EDC spectra (blue filled triangles), which gives confidence in this analysis. Interestingly, the slopes of both dispersions extrapolate to the same $k_{f}$ value, and the dispersion of the shoulder, $V_{\mathrm{sh}}=1.3 \mathrm{eV} \AA,{ }^{22}$ is close to the bare band value for $a_{1 g} .{ }^{20} \mathrm{We}$ conclude that both the narrow peak and the broad 

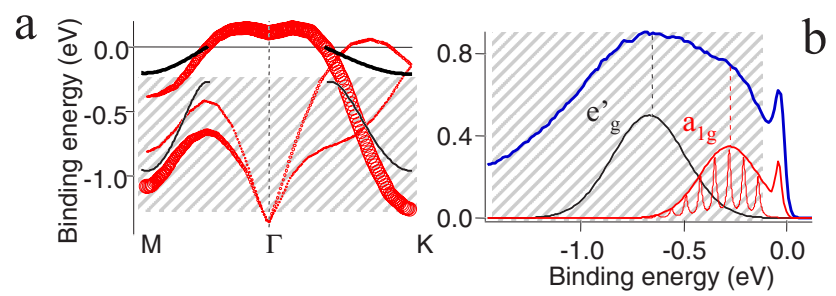

FIG. 3. (Color online) (a) Red circles: sketch of the band structure (from Ref. 23). The size of the marker is proportional to the $a_{1 g}$ character. Black lines: dispersion of the two components identified in Fig. 2. Shaded area corresponds to incoherent excitations. (b) Decomposition of a $\mathrm{BiBaCoO}$ spectrum near $E_{F}$ into $e_{g}^{\prime}$, and $a_{1 g}$ contributions. The $a_{1 g}$ line shape is sketched with a peak-dip-hump structure (see text).

shoulder belong to $a_{1 g}$. Figure 2(c) shows that the linewidth changes significantly in the two parts, increasing discontinuously from $30 \mathrm{meV}$ near $E_{F}$ to about $400 \mathrm{meV}$ in the shoulder. This further emphasizes the different natures of the two parts.

Within a band structure approach, a "break" in the dispersion could be due to the opening of a gap at the crossing between two bands. The similar two-peaks structure reported in $\mathrm{Na}$ cobaltates was indeed recently interpreted as resulting from a large hybridization gap between $a_{1 g}$ and $e_{g}^{\prime} .{ }^{9}$ The band structure calculated by LDA is recalled in Fig. 3(a), ${ }^{23}$ together with the dispersion of the two components extracted from Fig. 2(b) by a fit to cosine functions (we neglect here the small changes as a function of the direction in the reciprocal space ${ }^{9,21}$ ). Calculations predict the opening of a large hybridization gap between $a_{1 g}$ and $e_{g}^{\prime}$ along $\Gamma M, 20,23$ but not along $\Gamma K$, where it is forbidden by symmetry. ${ }^{20}$ As the line shape is essentially the same in the two directions, it is not likely that the hybridization gap is the origin of the structure. More fundamentally, this interpretation would not explain the change of dispersion and width in the two parts.

On the contrary, these features correspond, very well to a peak-dip-hump structure of the line shape, sketched in Fig. 3(b). The $a_{1 g}$ band would be formed by a leading QP peak and its incoherent tail or hump. The hump is represented in Fig. 3(b) as the envelope of multiple shake-up peaks corresponding, in a molecular picture, to different excited states. ${ }^{10}$ The QP peak follows by definition the band dispersion renormalized by the interactions, while the hump may follow the bare band dispersion. Whereas the width of the QP peak should correspond to the QP inverse lifetime, that of the hump is related to the structure of the incoherent excitations, and is typically much larger. These characteristics of dispersion and width perfectly fit our observations for the $a_{1 g}$ structure. Recently, very similar high-energy anomalies were reported in many strongly correlated systems. ${ }^{24-26}$ This structure therefore appears to be recurrent in correlated systems, although somewhat masked in cobaltates, because of the overlap with $e_{g}^{\prime}$.

Indeed, one can expect a contribution of the two $e_{g}^{\prime}$ bands in the low-energy part of the spectrum of Fig. 3(b). Its dispersion is not clearly observed in these experimental conditions. ${ }^{9}$ We briefly note that this interpretation of the line
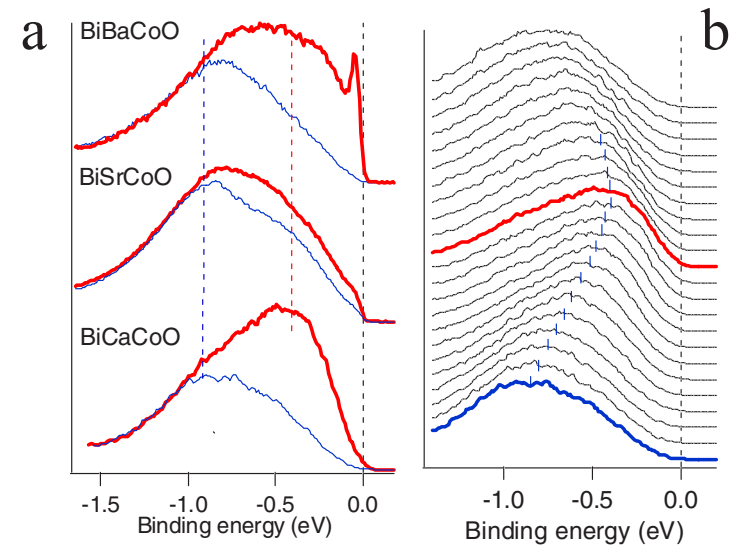

FIG. 4. (Color online) (a) Spectra at $20 \mathrm{~K}$ with minimum (thin blue line) and maximum (thick red line) weight at $E_{F}$ in $\mathrm{BiBaCoO}$ [see Fig. 2(a)], BiSrCoO, and BiCaCoO. (b) Dispersion of the hump in $\mathrm{BiCaCoO}$. Spectra are spaced by $0.063 \AA^{-1}$.

shape structure could have important consequences for the search for $e_{g}^{\prime}$ pockets in ARPES spectra. Reference 18 concluded that the pockets were pushed by correlations below $E_{F}$, to form a step at $-0.2 \mathrm{eV}$. This assignment is not as simple if there is a PDH structure of the line shape. As can be seen in Fig. 3(a), the $-0.2 \mathrm{eV}$ step could also be the bottom of the QP band, or possibly the top of an $e_{g}^{\prime}$ hump with a vanishingly small QP peak.

The central question raised by this study is the origin of the incoherent structure. Figure 4 reveals an evolution of the spectra through the Bi misfit family, which suggests answers. Figure 4(a) shows that the QP peak is strongly suppressed in $\mathrm{BiSrCoO}$ and disappears in $\mathrm{BiCaCoO}$. The qualitative trend of this evolution is maintained from 10 to $100 \mathrm{~K}$. A similar trend was recently reported in $\mathrm{Pb}$-doped $\mathrm{Bi}$ misfit phases. ${ }^{27}$ We further observe a dispersive hump, very similar to that observed in $\mathrm{BiBaCoO}$, as shown for $\mathrm{BiCaCoO}$ in Fig. 4(b). Spectra near the top and the bottom of the band are reported in red (thick line) and blue (thin line) on Fig. 4(a) to indicate that this dispersion occurs on a similar energy scale in the three compounds. Therefore, the nearly insulating nature of $\mathrm{BiCaCoO}$ (Ref. 6) does not result from a shift of the bands below $E_{F}$ but from a transfer of spectral weight from the QP peak to the hump. This behavior strongly supports the PDH interpretation, because the loss of weight at $E_{F}$ would be hardly understandable otherwise. ${ }^{15,28}$ The size of the QP peak relative to the hump is a measure of the strength of the interactions, pointing to larger many-body effects near the insulator.

In misfits with $\mathrm{Ba}, \mathrm{Sr}$, or $\mathrm{Ca}$, one expects the doping to change because of the different relative periodicities $m .^{13}$ Using the value $x=0.7$, determined by the present study for $\mathrm{Ba}$, we may estimate the effective doping for the other compounds by using the ratio of the periodicities. This yields $x$ $=0.8$ for $\mathrm{Sr}$ and $x=0.9$ for $\mathrm{Ca}$. We then interpret the evolution in Fig. 4 as the one toward the band insulator expected at $x=1$. In this limit, far from the Mott insulator, correlation effects should become negligible and the suppression of the QP peak is very puzzling. The paradox is similar to that stressed in the Introduction, with the appearance of large 
magnetic correlations in $\mathrm{Na}$ cobaltates near $x=1$. In $\mathrm{BiSrCoO}$ and $\mathrm{BiCaCoO}$, the magnetoresistance becomes large and negative, ${ }^{5}$ which also implies a strong coupling between carriers and magnetic excitations. It is then likely that magnetic excitations are a key ingredient for the PDH structure and define the nature of the metallic excitations in this limit. One possibility would be the "spin-orbital polarons" proposed by Daghofer et al. ${ }^{28}$ for $\mathrm{Na}_{x} \mathrm{CoO}_{2}$ near $x=1$. In this image, a hole (i.e., a $\mathrm{Co}^{4+}$ ion) distorts the surrounding $\mathrm{Co}^{3+}$ sites, allowing their excitation to high-spin states. This would create complex magnetic objects, with a spectral function markedly different from that of a simple QP. Dealing with elementary excitations extending over more than one site is also a possible way to solve the puzzle of the large correlations in the small-hole limit. Indeed, such "objects" would be stabilized, in the limit where they do not overlap too strongly.

To summarize, we have shown that the underlying electronic structure of misfit cobaltates is that of $\mathrm{CoO}_{2}$ slabs. In $\mathrm{BiBaCoO}$, a hexagonal FS is observed, as in $\mathrm{Na}$ cobaltates, the Fermi velocity is of the same order of magnitude, and the line shape structure is identical. The FS area corresponds to $x=0.7$ according to the Luttinger theorem. Increasing $x$ by replacing $\mathrm{Ba}$ with $\mathrm{Sr}$ or $\mathrm{Ca}$ leads to a strong suppression of the QP peak, never observed so far in Na cobaltates. We attribute this to a transfer of spectral weight toward an incoherent structure, which we identify as a broad hump dispersing at the bare band value, already present at lower $x$ values. This assignment is a step toward the understanding of correlation effects in cobaltates and suggests a parallel with other correlated systems. ${ }^{24}$ It favors a picture where elementary excitations are complex objects, formed by holes strongly coupled to magnetic and/or lattice excitations, and which possibly extend over more than one site. The overall lower conductivity of misfit phases with respect to $\mathrm{Na}$ phases, probably due to their larger disorder, suggests that these objects may localize at high $x$ and become more easily detectable.

We thank A. F. Santander-Syro for his contribution during the initial stage of this work, and H. Alloul, J. Bobroff, A. Georges, P. Mendels, F. Parmigiani, M. Rozenberg, and M. Shi for useful discussions.
${ }^{1}$ M. L. Foo, Y. Wang, S. Watauchi, H. W. Zandbergen, T. He, R. J. Cava, and N. P. Ong, Phys. Rev. Lett. 92, 247001 (2004).

${ }^{2}$ I. R. Mukhamedshin, H. Alloul, G. Collin, and N. Blanchard, Phys. Rev. Lett. 94, 247602 (2005).

${ }^{3}$ C. A. Marianetti and G. Kotliar, Phys. Rev. Lett. 98, 176405 (2007).

${ }^{4}$ M. Hervieu, A. Maignan, C. Michel, V. Hardy, N. Creon, and B. Raveau, Phys. Rev. B 67, 045112 (2003).

${ }^{5}$ T. Yamamoto, K. Uchinokura, and I. Tsukada, Phys. Rev. B 65, 184434 (2002).

${ }^{6}$ A. Maignan, S. Hebert, M. Hervieu, C. Michel, D. Pelloquin, and D. Khomskii, J. Phys.: Condens. Matter 15, 2711 (2003).

${ }^{7}$ M. Lee, L. Viciu, L. Li, Y. Wang, M. L. Foo, S. Watauchi, R. A. Pascal, Jr., R. J. Cava, and N. P. Ong, Nat. Mater. 5, 537 (2006).

${ }^{8}$ Y. Wang, N. S. Rogado, R. J. Cava, and N. P. Ong, Nature (London) 423, 425 (2003).

${ }^{9}$ D. Qian, L. Wray, D. Hsieh, L. Viciu, R. J. Cava, J. L. Luo, D. Wu, N. L. Wang, and M. Z. Hasan, Phys. Rev. Lett. 97, 186405 (2006).

${ }^{10}$ G. A. Sawatzky, Nature (London) 342, 480 (1989).

${ }^{11}$ H. Leligny, D. Grebille, O. Perez, A. C. Masset, M. Hervieu, and B. Raveau, Acta Crystallogr., Sect. B: Struct. Sci. 56, 173 (2000).

${ }^{12}$ In our single crystals, $\mathrm{K}$, for example, is found in the Ba-O planes $\left(\mathrm{K}_{2} \mathrm{CO}_{3}\right.$ or $\mathrm{KCl}$ is used as flux for the crystal growth).

${ }^{13}$ A. Maignan, D. Pelloquin, S. Hébert, Y. Klein, and M. Hervieu, in Proceedings of the 23rd International Conference on Thermoelectrics, Adelaide, 2004 (unpublished).
${ }^{14} \mathrm{~W}$. Kobayashi et al. (unpublished).

${ }^{15}$ T. Mizokawa, L. H. Tjeng, P. G. Steeneken, N. B. Brookes, I. Tsukada, T. Yamamoto, and K. Uchinokura, Phys. Rev. B 64, 115104 (2001).

${ }^{16}$ T. Takeuchi et al., Phys. Rev. B 69, 125410 (2004).

${ }^{17}$ T. Valla et al., Nature (London) 417, 627 (2002).

${ }^{18}$ H.-B. Yang et al., Phys. Rev. Lett. 95, 146401 (2005).

${ }^{19}$ D. Qian et al., Phys. Rev. Lett. 96, 216405 (2006).

${ }^{20}$ D. J. Singh, Phys. Rev. B 61, 13397 (2000).

${ }^{21}$ A. Nicolaou et al. (unpublished).

${ }^{22}$ To correct for the deviation from $\Gamma K$, this velocity should be divided by $\cos \theta=0.9$. We have found that such corrections work well within experimental accuracy for $\theta<50^{\circ}$.

${ }^{23}$ K.-W. Lee, J. Kuneš, and W. E. Pickett, Phys. Rev. B 70, 045104 (2004).

${ }^{24}$ N. Mannella, W. L. Yang, X. J. Zhou, H. Zheng, J. F. Mitchell, J. Zaanen, T. P. Devereaux, N. Nagaosa, Z. Hussain, and Z.-X. Shen, Nature (London) 438, 474 (2005).

${ }^{25}$ K. M. Shen et al., Phys. Rev. Lett. 93, 267002 (2004).

${ }^{26}$ F. Ronning, K. M. Shen, N. P. Armitage, A. Damascelli, D. H. Lu, Z.-X. Shen, L. L. Miller, and C. Kim, Phys. Rev. B 71, 094518 (2005); K. Yang et al., ibid. 73, 144507 (2006).

${ }^{27}$ Z. Yusof, B. O. Wells, T. Valla, P. D. Johnson, A. V. Fedorov, Q. Li, S. M. Loureiro, and R. J. Cava, arXiv:cond-mat/0610271 (unpublished).

${ }^{28}$ M. Daghofer, P. Horsch, and G. Khaliullin, Phys. Rev. Lett. 96, 216404 (2006) 